\title{
Plasma Cyclophilin A Is a Novel Biomarker for Coronary Artery Disease
}

\author{
Kimio Satoh, MD, PhD; Yoshihiro Fukumoto, MD, PhD; Koichiro Sugimura, MD, PhD; \\ Yutaka Miura, MD, PhD; Tatsuo Aoki, MD, PhD; Kotaro Nochioka, MD, PhD; \\ Shunsuke Tatebe, MD; Saori Miyamichi-Yamamoto, MD, PhD; Toru Shimizu, MD; \\ Shizuka Osaki, BSc; Yusuke Takagi, MD, PhD; Ryuji Tsuburaya, MD, PhD; \\ Yoshitaka Ito, $\mathrm{MD}, \mathrm{PhD}$; Yasuharu Matsumoto, $\mathrm{MD}, \mathrm{PhD}$; Masaharu Nakayama, $\mathrm{MD}, \mathrm{PhD}$; \\ Morihiko Takeda, MD, PhD; Jun Takahashi, MD, PhD; Kenta Ito, MD, PhD; \\ Satoshi Yasuda, MD, PhD; Hiroaki Shimokawa, MD, PhD
}

\begin{abstract}
Background: Oxidative stress induces secretion of cyclophilin A (CyPA) from vascular smooth muscle cells and it plays a crucial role in the pathogenesis of atherosclerosis in mice. Therefore, we tested our hypothesis that plasma CyPA levels are increased in patients with coronary artery diseases (CAD).

Methods and Results: In 320 consecutive patients undergoing coronary angiography, we examined the relationship between plasma CyPA levels and the severity of CAD. We measured plasma CyPA by an immunoassay based on the sandwich technique. Plasma CyPA levels were significantly higher in patients with significant coronary stenosis compared to those without it $(\mathrm{P}<0.001)$. A positive correlation was noted between plasma CyPA levels and significant coronary stenosis. The average number of stenotic coronary arteries and the need for coronary intervention were significantly increased in the quartiles of higher CyPA levels (both $\mathrm{P}<0.001$ ). Indeed, the plasma CyPA level significantly correlated with the presence of CAD (adjusted odds ratio for CAD, 6.20; 95\% confidence interval, 3.14-12.27; $P<0.001)$. Interestingly, plasma levels of CyPA increased according to the number of atherosclerotic risk factors, all of which induce oxidative stress. Furthermore, plasma levels of CyPA significantly reduced after medical treatment of risk factors. Finally, CyPA was strongly expressed in coronary atherosclerotic plaque in patients with myocardial infarction.
\end{abstract}

Conclusions: Plasma CyPA level is a novel biomarker for oxidative stress and CAD in humans. (Circ J 2013; 77: 447-455)

Key Words: Biomarker; Coronary artery disease; Oxidative stress

$\mathbf{T}$ he development of atherosclerosis is initiated by activation of endothelial cells (EC), leading to expression of adhesion molecules for inflammatory cells. ${ }^{1}$ A critical step in the progression of atherosclerosis is the development of an oxidizing environment because of the activation of macrophages that have become loaded with oxidized lowdensity lipoprotein (LDL) and other lipids. These macrophages produce abundant reactive oxygen species (ROS) and secrete several growth factors that contribute to the progression of atherosclerosis. ${ }^{2}$ It has been widely recognized that oxidative stress, generated by excessive ROS, promotes coronary artery diseases (CAD). ${ }^{3}$ Moreover, ROS induce the secretion of cyclophilin A (CyPA) from vascular smooth muscle cells
(VSMC) $)^{4,5}$ and extracellular CyPA induces EC adhesion molecule expression and promotes VSMC proliferation and migration. ${ }^{6-8}$

\section{Editorial p 321}

CyPA is a ubiquitously distributed protein belonging to the immunophilin family, and is recognized as the intracellular receptor for the immunosuppressive drug cyclosporin. ${ }^{9} \mathrm{CyPA}$ possesses peptidyl-prolyl isomerase activity and plays an important role in protein folding and trafficking of extracellular signal-regulated kinase $1 / 2^{10}$ and apoptosis-inducing factor. ${ }^{11}$ Although initially CyPA was thought to function primarily as

Received June 21, 2012; revised manuscript received September 10, 2012; accepted September 18, 2012; released online November 8,

2012 Time for primary review: 12 days

Department of Cardiovascular Medicine, Tohoku University Graduate School of Medicine, Sendai, Japan

The Guest Editor for this article was Hiroyuki Daida, MD.

Mailing address: Hiroaki Shimokawa, MD, PhD, Professor and Chairman, Department of Cardiovascular Medicine, Tohoku University

Graduate School of Medicine, Sendai 980-8574, Japan. E-mail: shimo@ cardio.med.tohoku.ac.jp

ISSN-1346-9843 doi:10.1253/circj.CJ-12-0805

All rights are reserved to the Japanese Circulation Society. For permissions, please e-mail: cj@j-circ.or.jp 
an intracellular protein, recent studies have revealed that it can be secreted by cells in response to ROS. Extracellular CyPA is a potent leukocyte chemoattractant, ${ }^{12,13}$ and importantly, plasma CyPA is significantly increased in patients with inflammatory diseases such as rheumatoid arthritis. ${ }^{14}$ Furthermore, we have found that CyPA expression in mice is closely associated with the development of intimal thickening, aortic aneurysms, and atherosclerosis. ${ }^{8,15-17}$ The secretion of CyPA is regulated by activation of Rho-kinase, ${ }^{7}$ which plays a crucial role in inflammation, vascular contraction, and the development of atherosclerosis. ${ }^{18,19}$ Thus, it seems plausible that the plasma levels of CyPA may discriminate between subjects at high or low risk for $\mathrm{CAD}$.

An ELISA assay for CyPA has recently been developed, enabling measurement of the plasma levels of CyPA. In the present study, we tested our hypothesis that circulating CyPA is detectable in patients with CAD and that these levels are associated with the number of atherosclerosis risk factors, the severity of CAD and the need for future cardiovascular intervention.

\section{Methods}

\section{Study Patients}

We conducted a prospective observational study of the prognostic value of CyPA in patients with symptoms or signs of CAD who were referred to the Tohoku University Hospital in Sendai for elective coronary angiography (CAG) from November 2007 through October 2011. If patients underwent angiography more than once, our analysis was based only on data obtained at the time of the first angiographic study. Patients with valvular or congenital heart disease were excluded. A total of 320 consecutive patients who had angina pectoris and evidence of ischemia on exercise ECG or myocardial radionuclide imaging were enrolled. Patients with unstable angina or myocardial infarction (MI) were excluded. The institutional Ethical Review Board approved the study, and written informed consent was given by all participating patients.

\section{Coronary Angiography}

At baseline, selective CAG was performed with recordings on the angiographic data system. Two experienced cardiologists, who were blinded to the patients' CyPA plasma levels, evaluated the angiograms. The degree of coronary stenosis was assessed in the direction that showed the most severe stenosis according to the American Heart Association standards. A narrowing of the lumen by more than $51 \%$ of the diameter was considered to indicate clinically significant stenosis. The patients were classified according to the severity of CAD as having no clinically significant organic stenosis, or 1-, 2-, or 3 -vessel disease. The left anterior descending coronary artery, left circumflex coronary artery, and right coronary artery were examined to evaluate the number of stenotic coronary arteries as 0 to 3 -vessel disease. Stenosis of the left main coronary artery was evaluated as 2-vessel disease. The relationship between the plasma CyPA level and the number of stenotic coronary arteries was analyzed using the average number of stenotic arteries to assess the severity of CAD.

\section{Immunostaining}

We have described the CyPA immunostaining in detail elsewhere. ${ }^{6-8}$ In brief, paraformaldehyde-fixed frozen sections were incubated overnight at $4^{\circ} \mathrm{C}$ with primary antibody ${ }^{20}$ (CyPA polyclonal, 1:1,000 dilution; BIOMOL Research Laboratories). For detection of CyPA, peroxidase-conjugated streptavidin
(1:1,000 dilution; Jackson Immuno) and NovaRed substrate kit (SK-4800, Vector Laboratories, Burlingame, CA, USA), were used and counterstained with hematoxylin. As a negative control, species- and isotype-matched IgG were used in place of the primary antibody.

\section{Baseline Measurements}

Information on vital status and data were obtained from the department's database system by means of a computerized search performed on December 7, 2011.21 No patients were lost to follow-up and all patients had a recorded medical history that included details of any previous MI, previous revascularization, angina pectoris, hypertension, previous stroke or transient ischemic attacks, diabetes, and smoking status. The cardiovascular risk was assessed in terms of hypertension, diabetes, smoking, aging and dyslipidemia. Patients with hypertension were assessed as being at risk if their blood pressure was $\geq 140 / 90 \mathrm{mmHg}$ or they had a history of antihypertensive drug use. Patients with diabetes mellitus were assessed as being at risk if their fasting glucose level was $\geq 126 \mathrm{mg} / \mathrm{dl}$ or they had a history of hypoglycemic drug or insulin use. Patients with dyslipidemia were assessed as being at risk if their LDL cholesterol was $\geq 140 \mathrm{mg} / \mathrm{dl}$ or their high-density lipoprotein cholesterol was $\leq 40 \mathrm{mg} / \mathrm{dl}$, or they were taking a lipidlowering drug. Before CAG, fasting blood samples for the measurement of CyPA were drawn from the antecubital vein of the patients who were resting supine. Plasma samples were collected using EDTA and centrifuged for $10 \mathrm{~min}$ at $2,500 \mathrm{~g}$ within $30 \mathrm{~min}$ of collection, and aliquots were stored at $-80^{\circ} \mathrm{C}$. CyPA was measured with use of an immunoassay based on the sandwich technique according to the protocol (Human Cyclophilin A ELISA Kit, CSB-E09920h, Cusabio). The detection limit was $0.78 \mathrm{ng} / \mathrm{ml}$. Across the entire analysis, duplicate measures of plasma CyPA level were highly correlated $(\mathrm{r}=0.92$, Figure S1A). The values of high-sensitivity CRP (hsCRP) were measured with a sandwich technique (Roche Diagnostics). The values of other laboratory parameters were obtained from samples assayed in an autoanalyzer in the hospital.

\section{Statistical Analysis}

Baseline characteristics of the study patients, grouped according to quartiles of $\mathrm{CyPA}$, are presented as frequencies and percentages, and continuous variables as means and standard deviations, or medians and interquartile ranges for variables with skewed distributions. Baseline characteristics were compared among quartiles with use of the chi square test for discrete variables and the Wilcoxon or Kruskal-Wallis rank-sum test for continuous variables when appropriate.

Additional CyPA analyses were performed in subgroups defined according to the results of angiography. A Student's ttest was used for comparisons between 2 groups and Dunnett's multiple comparison of means was used for multi-group comparison after analysis of variance (ANOVA). Receiver-operating-characteristic (ROC) curves were constructed to assess the sensitivity and specificity of plasma CyPA measurements obtained before CAG and to compare the ability to diagnose the existence and severity of CAD.

Logistic regression was used to estimate the association between plasma CyPA levels and CAD status after adjustment for age, sex, smoking status, presence or absence of diabetes, presence or absence of hypertension, and LDL cholesterol level. hsCRP levels were added in subsequent models. Adjusted odds ratios (ORs) are reported both for plasma CyPA levels $>15 \mathrm{ng} / \mathrm{ml}$ and across quartiles. Model performance was as- 

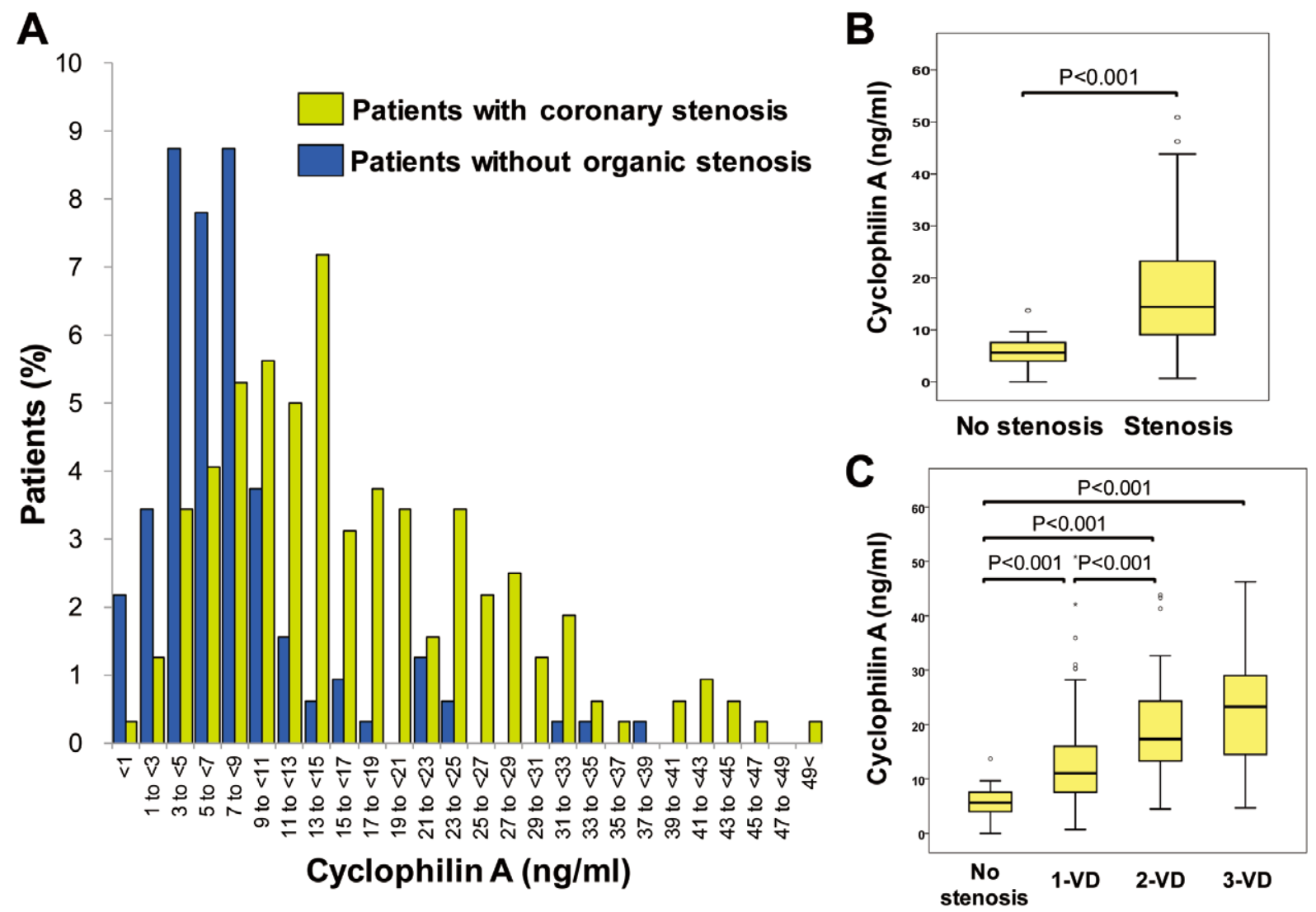

Figure 1. Circulating cyclophilin A (CyPA) levels in patients with 1-, 2-, or 3-vessel disease. (A) Green bars represent the number of patients with coronary artery stenosis and blue bars that of those without it. (B) Data are shown as box-and-whisker plots of CyPA in patients with organic stenosis $(>51 \%, n=189)$ and patients with no organic stenosis $(n=131)$. CyPA was elevated in patients with coronary stenosis $(\mathrm{P}<0.001)$ compared with patients without stenosis. (C) CyPA was elevated in patients with 1-, 2-, or 3 -vessel disease (all $\mathrm{P}<0.001$ ) compared with the control group (no organic stenosis/no vasospastic angina). CyPA increased sequentially within the coronary stenosis group as the number of stenotic vessels increased ( $P$ value for trend $<0.001)$.

sessed according to discrimination, by means of the area under the ROC (c-statistic); calibration, as indicated by the HosmerLemeshow goodness-of-fit statistic. ${ }^{22}$ Analyses were performed with CyPA as a categorical variable with the lowest quartile serving as reference for the other 3 quartiles. All reported $\mathrm{P}$ values are 2-tailed, with $\mathrm{P}<0.05$ indicating statistical significance. Analyses were performed with SPSS, version 19.0 (Chicago, IL, USA) and JMP, version 9.02 (Cary, NC, USA).

\section{Results}

\section{Plasma Levels of CyPA and Angiographic Status}

Figure 1A shows the distribution of patients with and without coronary artery stenosis by the plasma level of CyPA. The plasma levels of CyPA were significantly higher in patients with coronary organic stenosis compared with those without stenosis (Figure 1B). Moreover, the CyPA level increased with the severity of angiographic CAD $(\mathrm{P}<0.001$, Figure $1 \mathrm{C})$.

All the cases were divided into quartile groups based on the plasma level of CyPA to examine its correlation with the number of stenotic coronary arteries. Table 1 shows the patients' clinical background and laboratory data according to the quartiles of CyPA. Patients with CyPA in the upper quartile were older and were more likely to have clinically significant CAD $(\mathrm{P}<0.001$, Table 1$)$. The prevalence of both hypertension and diabetes was higher in the $4^{\text {th }}$ quartile, and these patients showed a slightly reduced estimated glomerular filtration rate (eGFR). The number of stenotic coronary arteries was significantly increased in the higher quartiles of CyPA $(\mathrm{P}<0.001$, Figure 2A). Furthermore, the requirement for cardiovascular intervention, such as percutaneous coronary intervention (PCI) and coronary artery bypass grafting $(\mathrm{CABG})$, was significantly increased in the $4^{\text {th }}$ quartile compared with the lower quartiles $(\mathrm{P}<0.001$, Figure 2B). We calculated the ROC curves and cstatistic on the basis of the plasma CyPA levels. The ROC curves demonstrated that the plasma level of CyPA is useful for the diagnosis of coronary organic stenosis (c-statistic=0.802) and the requirement for cardiovascular intervention (c-statistic $=0.793)$ (Figures 2C,D).

\section{Plasma Levels of CyPA and the Severity of CAD}

CyPA was elevated in patients with traditional cardiovascular risk factors such as hypertension, diabetes, smoking, dyslipidemia and advanced age (all $\mathrm{P}<0.001$, Figure 3 ). Division of the cohort into quartiles according to plasma CyPA level provided additional evidence of an association between plasma 


\begin{tabular}{|c|c|c|c|c|c|}
\hline & $\begin{array}{l}1^{\text {st }} \text { quartile } \\
\quad(n=80)\end{array}$ & $\begin{array}{l}2^{\text {nd }} \text { quartile } \\
\qquad(n=80)\end{array}$ & $\begin{array}{l}3^{\text {rd }} \text { quartile } \\
\quad(n=80)\end{array}$ & $\begin{array}{l}4^{\text {th }} \text { quartile } \\
\quad(n=80)\end{array}$ & $P$ value \\
\hline CyPA level (ng/ml) & $0.0-6.1$ & $6.2-9.6$ & $9.7-17.4$ & $17.5-50.9$ & \\
\hline Age (years) & & & & & $<0.001$ \\
\hline Median & 58 & 63 & 68 & 68 & \\
\hline Interquartile range & $50-68$ & $55-71$ & $60-75$ & $60-74$ & \\
\hline Male sex (\%) & 60 & 61 & 66 & 76 & 0.122 \\
\hline Family history of IHD (\%) & 11 & 18 & 4 & 10 & 0.046 \\
\hline \multicolumn{6}{|l|}{ Medical history (\%) } \\
\hline Hypertension & 56 & 59 & 75 & 88 & $<0.001$ \\
\hline Diabetes & 36 & 34 & 48 & 55 & 0.022 \\
\hline Dyslipidemia & 51 & 51 & 70 & 80 & $<0.001$ \\
\hline Current smoker (\%) & 28 & 34 & 49 & 59 & $<0.001$ \\
\hline Angiographic findings (\%) & & & & & $<0.001$ \\
\hline No coronary artery stenosis & 71 & 61 & 20 & 13 & \\
\hline 1-vessel disease & 23 & 31 & 41 & 29 & \\
\hline 2-vessel disease & 3 & 4 & 24 & 31 & \\
\hline 3-vessel disease & 4 & 4 & 11 & 28 & \\
\hline $\begin{array}{l}\text { Requirement for } \mathrm{PCl} \text { or } \mathrm{CABG} \text { during } \\
\text { follow-up }(\%)\end{array}$ & 8 & 8 & 23 & 40 & 0.019 \\
\hline $\mathrm{BMI}^{*}$ & & & & & 0.003 \\
\hline Median & 25 & 24 & 24 & 23 & \\
\hline Interquartile range & $23-27$ & $22-26$ & $21-26$ & $21-25$ & \\
\hline eGFR $\left(\mathrm{ml} \cdot \mathrm{min}^{-1} \cdot 1.73 \mathrm{~m}^{-2}\right)$ & $54 \pm 17$ & $59 \pm 19$ & $51 \pm 20$ & $49 \pm 19$ & $<0.001$ \\
\hline Left ventricular ejection fraction (\%) & $65 \pm 10$ & $66 \pm 8$ & $59 \pm 12$ & $63 \pm 12$ & 0.090 \\
\hline \multicolumn{6}{|l|}{ Lipid status (mg/dl) } \\
\hline LDL cholesterol & $105 \pm 36$ & $105 \pm 29$ & $96 \pm 35$ & $97 \pm 33$ & 0.078 \\
\hline HDL cholesterol & $47 \pm 13$ & $49 \pm 12$ & $49 \pm 12$ & $49 \pm 14$ & 0.837 \\
\hline Triglycerides & $127 \pm 69$ & $147 \pm 95$ & $130 \pm 73$ & $132 \pm 100$ & 0.576 \\
\hline Hemoglobin $A_{1 c}$ & $6.4 \pm 1.4$ & $6.3 \pm 0.8$ & $6.4 \pm 0.9$ & $6.6 \pm 1.2$ & 0.067 \\
\hline hsCRP (mg/L) & $2.3 \pm 3.2$ & $1.7 \pm 2.6$ & $2.3 \pm 3.1$ & $1.8 \pm 2.8$ & 0.103 \\
\hline \multicolumn{6}{|l|}{ Medication (\%) } \\
\hline Aspirin & 16 & 30 & 51 & 71 & $<0.001$ \\
\hline$\beta$-blocker & 25 & 29 & 46 & 55 & $<0.001$ \\
\hline Statin & 39 & 35 & 56 & 70 & $<0.001$ \\
\hline ACE inhibitor & 28 & 24 & 31 & 39 & 0.212 \\
\hline ARB & 24 & 26 & 28 & 33 & 0.654 \\
\hline Calcium-channel blocker & 51 & 55 & 61 & 58 & 0.550 \\
\hline
\end{tabular}

Data are shown as mean \pm SD unless otherwise shown.

${ }^{*} \mathrm{BMl}=$ weight in $\mathrm{kg} /(\text { height in } \mathrm{m})^{2}$.

ACE, angiotensin-converting enzyme; ARB, angiotensin-receptor blocker; BMI, body mass index; CABG, coronary artery bypass grafting; CyPA, cyclophilin A; HDL, high-density lipoprotein; hsCRP, high-sensitivity C-reactive protein; IHD, ischemic heart disease; LDL, low-density lipoprotein; $\mathrm{PCl}$, percutaneous coronary intervention.

CyPA and CAD (Table 2). In the analysis adjusted for age, sex, and traditional cardiovascular risk factors (smoking, diabetes, hypertension, dyslipidemia), quartiles 2,3 , and 4 of plasma CyPA were associated with an increased risk of CAD as compared with the quartile of lowest CyPA (OR, 1.73, 9.94, and 10.29; P-value for trend $<0.001$ ). This result remained significant after adjustment for traditional cardiovascular risk factors plus hsCRP levels (OR, 1.84, 10.53, and 10.78; P-value for trend $<0.001$ ). Several known cardiovascular risk factors were associated with CAD in logistic-regression models adjusted for age, sex, and body mass index (BMI) (Figure 4). Diabetes and hypertension were each linked to an increased risk of CAD. Each of the known risk factors, in addition to plasma CyPA, was combined in a single logistic-regression analysis (Figure 4). In this model, which included the hsCRP level, plasma CyPA $>15 \mathrm{ng} / \mathrm{ml}$ remained highly related with disease status (OR 6.20, $\mathrm{P}<0.001)$. Multivariable analysis demonstrated that, in addition to the established risk factors (age, sex, smoking, hypertension, diabetes and hsCRP), CyPA $>15 \mathrm{ng} / \mathrm{ml}$ was significantly correlated with CAD (Table 3).

The inclusion of plasma CyPA resulted in significant improvement of the overall performance of the logistic-regression model. The c-statistic increased from 0.807 to 0.870 when plasma CyPA was added to known cardiovascular risk factors (age, sex, smoking, hypertension, diabetes, dyslipidemia). When the hsCRP level was included in the baseline model, the cstatistic increased from 0.807 to 0.873 . The addition of plasma CyPA did not reduce model discrimination as assessed by goodness-of-fit statistics. CyPA added prognostic information above and beyond that provided by age, sex, family history 


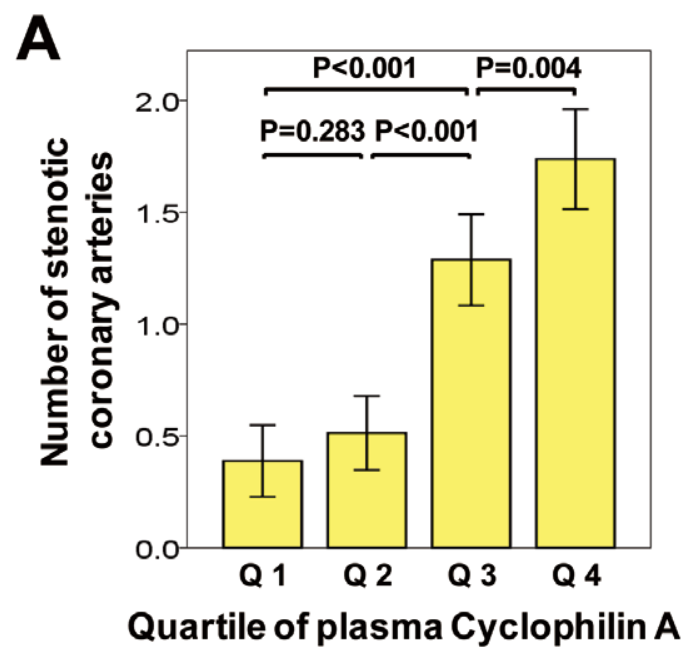

B

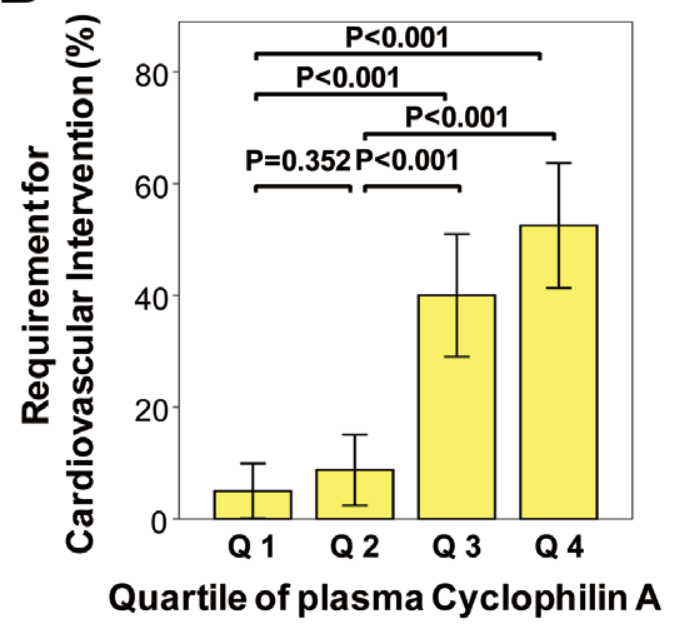

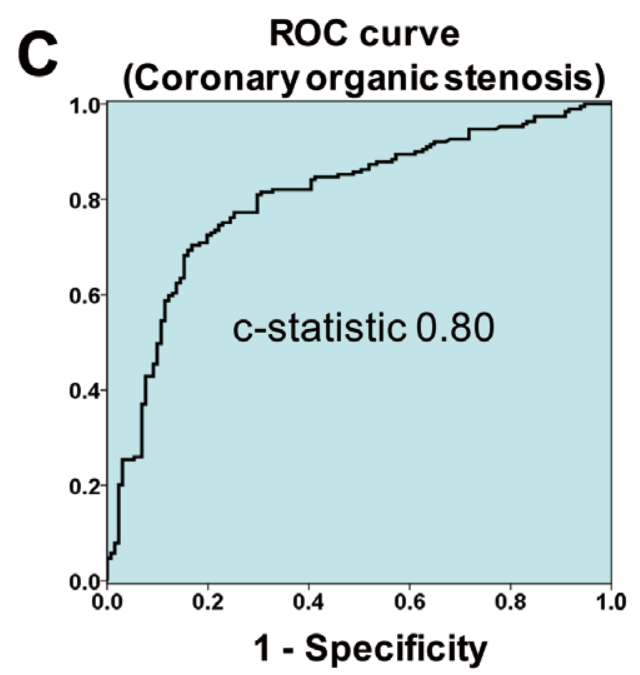

D

ROC curve (Cardiovascular intervention)

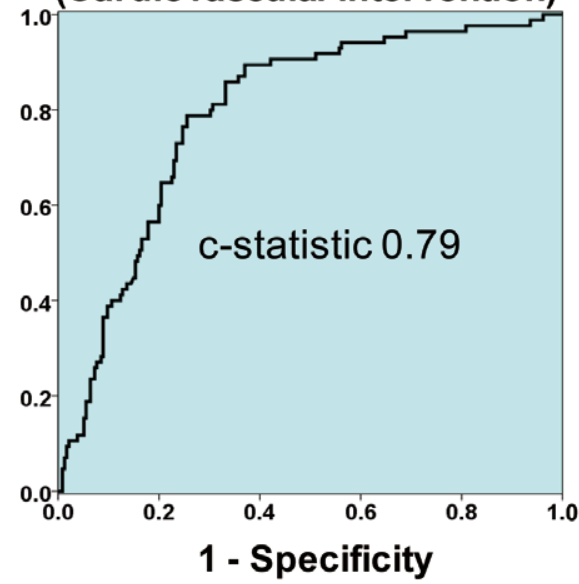

Figure 2. Number of stenotic coronary arteries and requirement for cardiovascular intervention according to quartiles of cyclophilin A (CyPA). Data from 320 patients with and without coronary stenosis are divided according to the quartiles of plasma CyPA levels. The CyPA levels were as follows: $1^{\text {st }}$ quartile (Q1), $<6.1 \mathrm{ng} / \mathrm{ml} ; 2^{\text {nd }}$ quartile (Q2), 6.2-9.6 $\mathrm{ng} / \mathrm{ml}$; $3^{\text {rd }}$ quartile (Q3), $9.7-17.4 \mathrm{ng} / \mathrm{ml} ; 4^{\text {th }}$ quartile $(\mathrm{Q} 4),>17.5 \mathrm{ng} / \mathrm{ml}$. P $<0.001$ by the log-rank test for the overall comparison among the groups. (A) Number of stenotic coronary arteries according to quartiles of CyPA. CyPA was elevated in patients in Q3 $(P<0.001)$ and Q4 $(P<0.001)$ compared with $Q 1$. The number of stenotic coronary arteries increased sequentially as the quartiles increased. $(B)$ Requirement for cardiovascular intervention according to quartiles of CyPA. The requirement was higher in patients with Q4 compared with Q1 $(P<0.001)$ and Q2 $(P<0.001)$. Cardiovascular intervention included percutaneous coronary intervention $(\mathrm{PCl})$ and coronary artery bypass grafting (CABG). (C,D) Receiver-operating-characteristic curves (ROC) and C-statistic for baseline measurements of CyPA. Also shown are the sensitivity and specificity of these measures. (C) ROC curve describing the diagnostic performance of CyPA to identify coronary organic stenosis $>50 \%$ in at least 1 vessel as compared with the reference standard of invasive quantitative coronary angiography (CAG). The c-statistic was 0.80 (95\% CI, 0.75-0.85). (D) ROC curve describing the diagnostic performance of CyPA to identify the requirement for future cardiovascular intervention as compared with the reference standard of invasive quantitative CAG. The c-statistic was $0.79(95 \% \mathrm{Cl}, 0.74-0.85)$. Cl, confidence interval.

with respect to ischemic heart disease, presence or absence of hypertension, diabetes, smoking status, BMI, eGFR, and plasma lipid level. Excluding the 141 patients with high hsCRP did not significantly change the results. In patients with hsCRP $<1.000$, the adjusted OR for CAD in the $4^{\text {th }}$ quartile of CyPA, as compared with the $1^{\text {st }}$ quartile, was 13.2 (95\% confidence interval, 3.2-53.9, P<0.001). Additionally, CyPA ( $>15 \mathrm{ng} / \mathrm{ml})$ remained a strong prognostic marker, with an adjusted OR of 5.9 (95\% confidence interval, $2.3-14.8, \mathrm{P}<0.001)$. Among pa- tients with hsCRP $>1.000$, the same trend was observed, suggesting the potential usefulness of combining these biomarkers for CAD.

\section{Plasma Levels of CyPA as a Biomarker of Therapeutic Outcome}

Interestingly, the plasma level of CyPA increased according to the atherosclerotic risk factors such as sex, hypertension, diabetes mellitus, dyslipidemia, and smoking, all of which are 


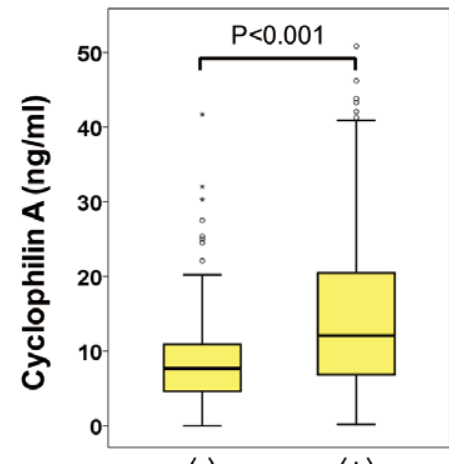

$(-) \quad(+)$

Hypertension

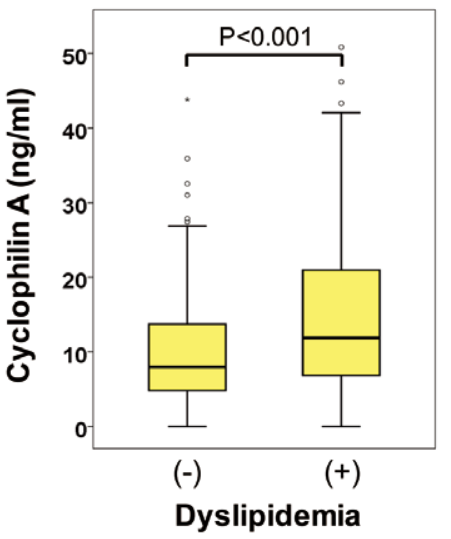

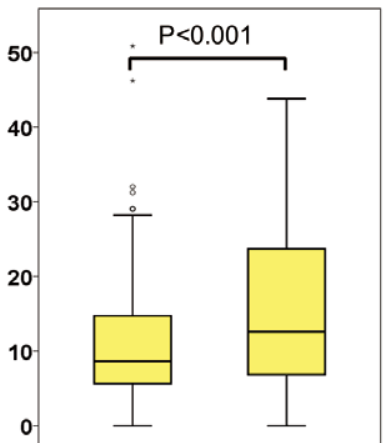

(+)

$(-)$ Diabetes

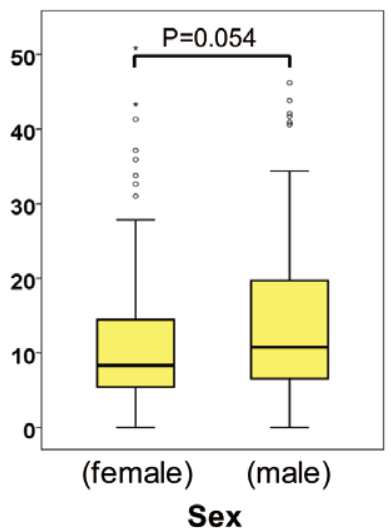

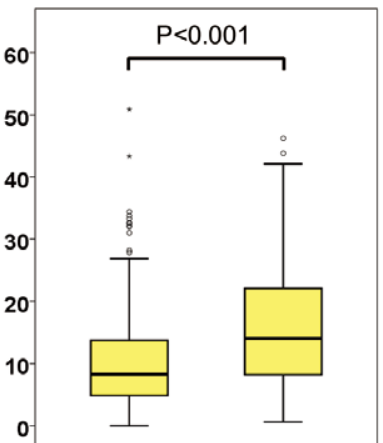

$(-)$

(+)

Smoking

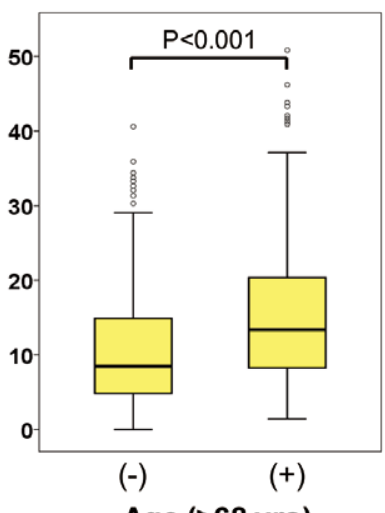

Age (>68 yrs)

Figure 3. Odds ratios for coronary artery disease according to plasma cyclophilin A (CyPA) level and selected risk factors. The logistic-regression model was also adjusted for age, sex, and body mass index. Odds ratios for continuous variables are per 1-SD increase.

\begin{tabular}{l} 
Table 2. CAD Status According to Quartiles of Plasma CyPA \\
\cline { 3 - 4 } \\
\cline { 3 - 4 }
\end{tabular}

${ }^{*}$ Cardiovascular risk factors included in the logistic-regression model were age, sex, smoking status, presence or absence of diabetes, presence or absence of hypertension, and presence or absence of dyslipidemia.

$\mathrm{CAD}$, coronary artery disease; $\mathrm{Cl}$, confidence interval; OR, odds ratio. Other abbreviations as in Table 1.

oxidative stress inducers (Figure 3). Therefore, plasma CyPA may be important as a biomarker of therapeutic outcome after controlling risk factors. To further confirm the role of plasma CyPA, we performed a follow-up study after the addition of drugs to control risk factors. After treatment in several individuals $(n=42)$, the plasma obtained at baseline and follow-up (mean follow-up, 273 days) revealed a significant reduction after treatment $(\mathrm{P}=0.003$, Figure 5A). Medical treatments that control atherosclerotic risk factors decreased plasma CyPA levels in patients with stable $\mathrm{CAD}$, suggesting that plasma CyPA is useful for the evaluation of systemic oxidative stress and the therapeutic effect of medication.

\section{CyPA and Atherosclerotic Unstable Plaque}

As demonstrated, ROS-induced secretory protein CyPA is a useful biomarker of CAD, so we hypothesized that secreted CyPA is highly accumulated in atherosclerotic plaque of coronary arteries. Indeed, we observed strong CyPA expression in coronary arteries in patients with MI (Figures 5B,C). Importantly, the strong expression of CyPA was localized just beneath the thin fibrous cap of atherosclerotic plaque. 


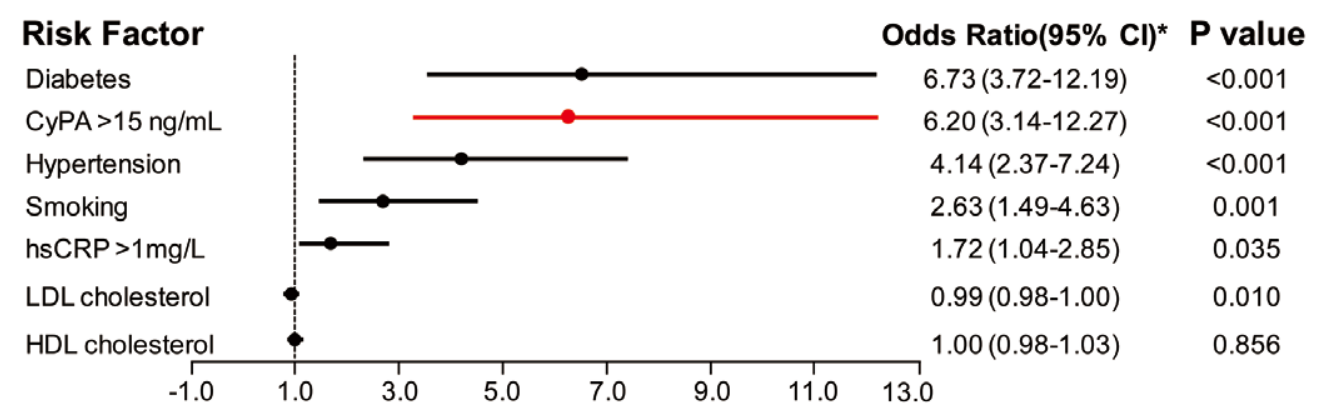

Figure 4. Plasma levels of cyclophilin A (CyPA) and cardiovascular risk factors. Plasma levels of CyPA were significantly increased by the presence of hypertension, diabetes, smoking, dyslipidemia and advanced age of 68 years or older but not by sex difference. *Adjusted with age, sex, and BMI.

\begin{tabular}{|c|c|c|c|c|c|c|}
\hline & \multicolumn{3}{|c|}{ Univariable analysis } & \multicolumn{3}{|c|}{ Multivariable analysis* } \\
\hline & OR & $95 \% \mathrm{Cl}$ & $P$ value & OR & $95 \% \mathrm{Cl}$ & $P$ value \\
\hline Age & 1.08 & $1.05-1.10$ & $<0.001$ & 1.07 & $1.04-1.10$ & $<0.001$ \\
\hline Men & 1.76 & $1.10-2.82$ & 0.018 & 1.58 & $0.81-3.09$ & 0.184 \\
\hline BMI & 0.95 & $0.89-1.01$ & 0.085 & & & \\
\hline Hypertension & 5.34 & $3.19-8.93$ & $<0.001$ & 1.85 & $0.95-3.63$ & 0.073 \\
\hline Smoking & 2.44 & $1.52-3.90$ & $<0.001$ & 1.82 & $0.95-3.49$ & 0.072 \\
\hline Dyslipidemia & 4.03 & $2.49-6.51$ & $<0.001$ & 2.37 & $1.23-4.55$ & 0.010 \\
\hline Diabetes & 5.87 & $3.50-9.86$ & $<0.001$ & 4.71 & $2.53-8.78$ & $<0.001$ \\
\hline Family history of IHD & 0.663 & $0.33-1.35$ & 0.258 & & & \\
\hline СуPA $>15 \mathrm{ng} / \mathrm{ml}$ & 7.58 & $4.00-14.38$ & $<0.001$ & 4.14 & $2.00-8.57$ & $<0.001$ \\
\hline hsCRP > $1 \mathrm{mg} / \mathrm{L}$ & 1.77 & $1.12-2.79$ & 0.014 & 1.58 & $0.87-2.86$ & 0.135 \\
\hline
\end{tabular}

${ }^{*}$ Analysis was performed on 8 variables including age, sex, hypertension, smoking, dyslipidemia, diabetes mellitus, CyPA $>15 \mathrm{ng} / \mathrm{ml}$ and $\mathrm{hsCRP}>1 \mathrm{mg} / \mathrm{L}$.

Abbreviations as in Tables 1,2.

\section{Discussion}

Our study demonstrated that the plasma level of CyPA in patients with stable CAD provides prognostic information on the severity of CAD and the requirement for cardiovascular intervention. The findings of the present study support our previous results in mice suggesting that CyPA augments the development of atherosclerosis. ${ }^{8,17,23}$ Patients with high CyPA levels had a significantly higher prevalence of CAD on CAG than those with low levels of CyPA. A possible role for CyPA in atherosclerosis is becoming increasingly apparent. We have shown that knock-down of CyPA in EC reduced apoptosis induced in vitro by tumor-necrosis factor- $\alpha$ and that CyPA deficiency was associated with a marked decrease in EC apoptosis in the early stages of atherosclerosis. ${ }^{17}$ The increase in vascular oxidative stress requires $\mathrm{CyPA}^{15}$ which thereby sensitizes $\mathrm{EC}$ to apoptosis. In addition, CyPA secretion is regulated by Rho-kinase activation, which is important for VSMC contraction and atherosclerosis. ${ }^{19}$ Consistently, plasma levels of CyPA were significantly increased in patients with CAD.

In the present study, plasma levels of CyPA were elevated in patients with angiographically verified coronary atherosclerosis. We have previously demonstrated that ROS inducers, such as mechanical stress, angiotensin II and dyslipidemia, promote the secretion of CyPA ${ }^{8,15,17}$ in a Rho-kinase-dependent manner. ${ }^{19,24}$ It is well known that Rho-kinase is associated with activation of the NADPH oxidases, with resultant ROS production, ${ }^{25}$ which plays a crucial role in the development of several cardiovascular diseases. In support of this notion, CyPA was elevated in patients with hypertension, diabetes, smoking, dyslipidemia, and advanced age in the present study. This is the first study that has examined the association between CyPA and ROS inducers, all of which are atherosclerotic risk factors in humans.

\section{Plasma CyPA and Atherosclerotic Risk Factors}

In the present study, we further examined the prognostic importance of CyPA in patients with stable CAD. We found that CyPA is a prognostic marker for requirement of cardiovascular intervention such as PCI and CABG. The increased severity of CAD we observed among patients with elevated CyPA may be a consequence of a higher frequency of risk factors for atherosclerosis, all of which promote ROS production and CyPA secretion. All these mechanisms, while promoting an environment of oxidative stress, are likely to contribute to the increased plasma levels of CyPA in patients with severe CAD. Vascular ROS formation can be stimulated by mechanical stretch, pressure, shear stress, environmental factors such as hypoxia, and secreted factors such as angiotensin II. $^{27-31}$ In addition, extracellular CyPA induces ROS production in VSMC 

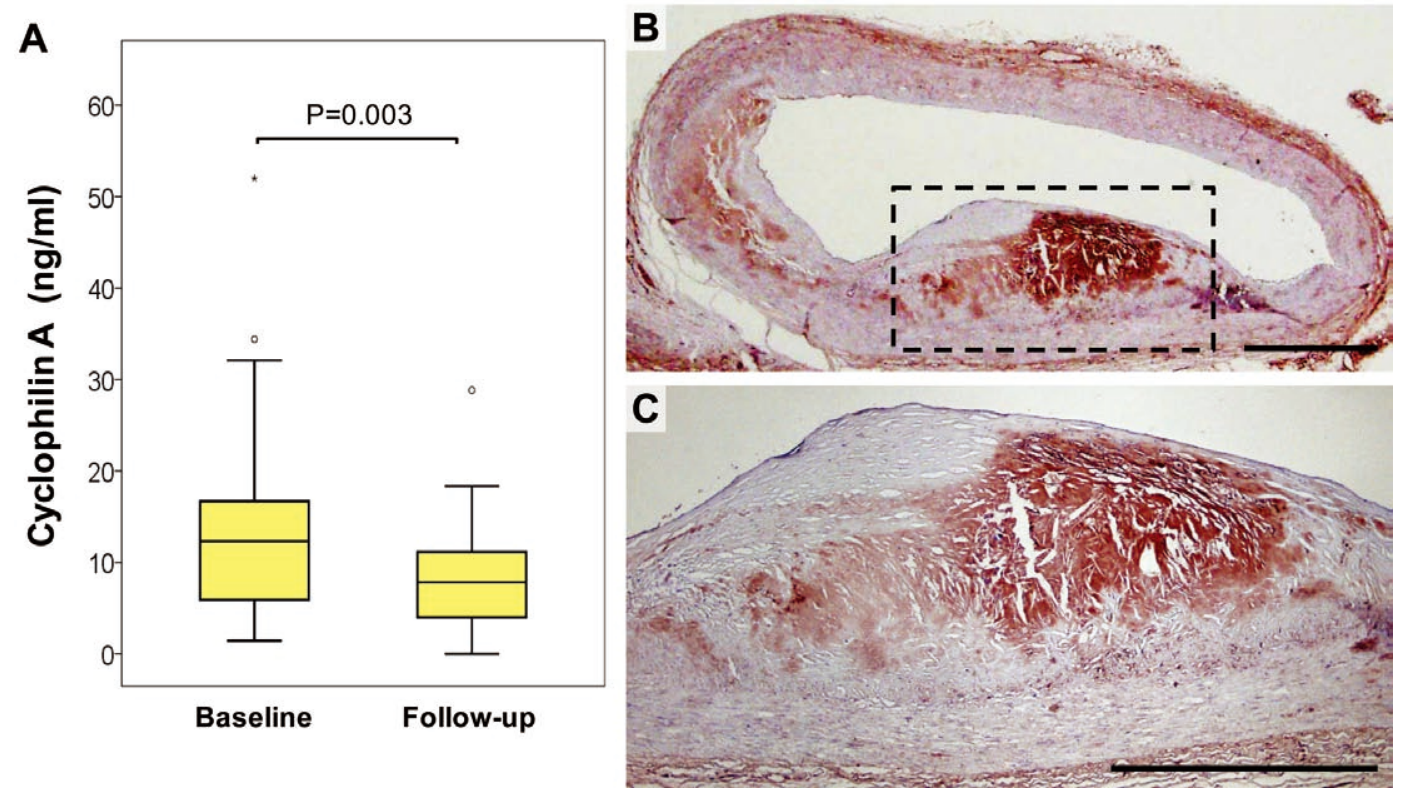

Figure 5. Plasma levels of cyclophilin A (CyPA) after treatment and representative immunostaining for CyPA in atherosclerotic coronary plaque from a patient with acute myocardial infarction. (A) Plasma levels of CyPA at baseline and follow-up ( $n=42$, mean follow-up, 273 days). (B,C) Coronary artery sample obtained from a patient with acute myocardial infarction showing strong CyPA expression just beneath the thin fibrous cap of atherosclerotic plaque. Bars $=500 \mu \mathrm{m}$.

and recruitment of inflammatory cells, resulting in augmentation of vascular ROS and atherosclerosis. ${ }^{17}$ All these data comprise a proof-of-concept that circulating CyPA is a novel biomarker for CAD and also plays a crucial role in ROS augmentation. Several risk factors, such as hypertension, diabetes, smoking and aging, induce the generation of ROS and promote the secretion of CyPA. Circulating CyPA augments ROS production synergistically. ${ }^{24}$ Therefore, secreted plasma CyPA, acting as a pro-inflammatory cytokine, synergistically augments ROS production, contributing to the onset of atherosclerosis and its progression. ${ }^{32}$

\section{CyPA and Atherosclerotic Unstable Plaque}

CyPA may play an important role in several stages of atherosclerosis. During fatty streak formation, it may play a role in lipid uptake via its effect on scavenger receptors. ${ }^{17}$ In all stages, it may play a role in inflammation by promoting monocyte adhesion and recruitment, as well as by contributing to an oxidative environment. The data from Seizer et al that CyPA is secreted from foam cells suggest an important role in the later stages of atherosclerosis. ${ }^{33}$ Indeed, we observed strong CyPA expression in the atherosclerotic plaque of patients with MI. Altogether, our data suggest that the agents that inhibit CyPA secretion might be candidates for suppressing the development of atherosclerosis. EMMPRIN, a putative CyPA receptor, has been identified as a tumor cell membrane protein that is expressed in VSMC, is activated by ROS and stimulates matrix metalloproteinase production. ${ }^{34} \mathrm{~A}$ recent study demonstrated ROS-dependent increases in EMMPRIN, ${ }^{35}$ which may be activated by the binding of extracellular CyPA. ${ }^{36}$ Therefore, ROS-induced secretion of CyPA may also contribute to the development of unstable atherosclerotic plaque and plaque rupture. We consider that the discovery of more selective and specific inhibitors of CyPA secretion may be an effective therapeutic approach for CAD.

\section{Study Limitations}

Several limitations should be mentioned. First, the study population was relatively small. However, even in this small population, plasma levels of CyPA closely related with the severity of CAD. Future analysis in a large population prospective cohort will further elucidate the importance of plasma CyPA in CAD. Second, as to the plasma levels of CyPA in patients with MI, we need to consider 2 different mechanisms that increase the plasma level of CyPA. One mechanism is oxidative stress-induced CyPA secretion from the vasculature and another is CyPA release from necrotic tissue after MI. Therefore, in the present study, we excluded patients with unstable CAD or $\mathrm{MI}$ and recruited patients with stable CAD. The plasma levels in patients with unstable CAD or MI need to be examined in future studies.

In conclusion, the present study indicates that the plasma CyPA level is a novel biomarker of CAD. Further studies are needed to further establish the clinical significance of CyPA in the pathogenesis of atherosclerotic cardiovascular disease.

\section{Acknowledgments}

This work was supported in part by a Grant-in-Aid for Scientific Research on Innovative Areas (Signaling Functions of Reactive Oxygen Species) (08107961), a Grant-in-Aid for Tohoku University Global COE for Conquest of Signal Transduction Diseases with Network Medicine, and Grantsin-Aid for Scientific Research (08101562, 09006924 and 10002019), all of which are from the Ministry of Education, Culture, Sports, Science and Technology, Tokyo, Japan and the Grants-in-Aid for Scientific Research from the Ministry of Health, Labour, and Welfare, Tokyo, Japan (10102895), 
Japan Heart Foundation Research Grant, Mitsui Life Social Welfare Foundation and AstraZeneca Research Grant. We are grateful to the laboratory staff in the Department of Cardiovascular Medicine at the Tohoku University for their useful suggestions and technical assistance, especially that performed by Akemi Saito and Naomi Yamaki.

\section{Disclosures}

Conflict of Interest: None declared.

\section{References}

1. Berk BC. Atheroprotective signaling mechanisms activated by steady laminar flow in endothelial cells. Circulation 2008; 117: 1082-1089.

2. Hansson GK. Inflammation, atherosclerosis, and coronary artery disease. N Engl J Med 2005; 352: 1685-1695.

3. Dzau VJ, Antman EM, Black HR, Hayes DL, Manson JE, Plutzky J, et al. The cardiovascular disease continuum validated: Clinical evidence of improved patient outcomes. Part I: Pathophysiology and clinical trial evidence (risk factors through stable coronary artery disease). Circulation 2006; 114: 2850-2870.

4. Jin ZG, Melaragno MG, Liao DF, Yan C, Haendeler J, Suh YA, et al. Cyclophilin $\mathrm{A}$ is a secreted growth factor induced by oxidative stress. Circ Res 2000; 87: 789-796.

5. Liao DF, Jin ZG, Baas AS, Daum G, Gygi SP, Aebersold R, et al. Purification and identification of secreted oxidative stress-induced factors from vascular smooth muscle cells. J Biol Chem 2000; 275: $189-196$.

6. Jin ZG, Lungu AO, Xie L, Wang M, Wong C, Berk BC. Cyclophilin $\mathrm{A}$ is a proinflammatory cytokine that activates endothelial cells. Arterioscler Thromb Vasc Biol 2004; 24: 1186-1191.

7. Suzuki J, Jin ZG, Meoli DF, Matoba T, Berk BC. Cyclophilin A is secreted by a vesicular pathway in vascular smooth muscle cells. Circ Res 2006; 98: 811-817.

8. Satoh K, Matoba T, Suzuki J, O’Dell MR, Nigro P, Cui Z, et al. Cyclophilin A mediates vascular remodeling by promoting inflammation and vascular smooth muscle cell proliferation. Circulation 2008; 117: $3088-3098$.

9. Handschumacher RE, Harding MW, Rice J, Drugge RJ, Speicher DW. Cyclophilin: A specific cytosolic binding protein for cyclosporin A. Science 1984; 226: 544-547.

10. Pan H, Luo C, Li R, Qiao A, Zhang L, Mines M, et al. Cyclophilin $\mathrm{A}$ is required for CXCR4-mediated nuclear export of heterogeneous nuclear ribonucleoprotein A2, activation and nuclear translocation of ERK1/2, and chemotactic cell migration. J Biol Chem 2008; 283: $623-637$.

11. Zhu C, Wang X, Deinum J, Huang Z, Gao J, Modjtahedi N, et al. Cyclophilin A participates in the nuclear translocation of apoptosisinducing factor in neurons after cerebral hypoxia-ischemia. $J$ Exp Med 2007; 204: 1741-1748.

12. Arora K, Gwinn WM, Bower MA, Watson A, Okwumabua I, MacDonald HR, et al. Extracellular cyclophilins contribute to the regulation of inflammatory responses. J Immunol 2005; 175: $517-$ 522.

13. Damsker JM, Bukrinsky MI, Constant SL. Preferential chemotaxis of activated human CD4+ T cells by extracellular cyclophilin A. $J$ Leukoc Biol 2007; 82: 613-618.

14. Kim H, Kim WJ, Jeon ST, Koh EM, Cha HS, Ahn KS, et al. Cyclophilin A may contribute to the inflammatory processes in rheumatoid arthritis through induction of matrix degrading enzymes and inflammatory cytokines from macrophages. Clin Immunol 2005; 116: $217-224$.

15. Satoh K, Nigro P, Matoba T, O’Dell MR, Cui Z, Shi X, et al. Cyclophilin A enhances vascular oxidative stress and the development of angiotensin II-induced aortic aneurysms. Nat Med 2009; 15: 649656.

16. Weintraub NL. Understanding abdominal aortic aneurysm. $N$ Engl $J$ Med 2009; 361: 1114-1116.

17. Nigro P, Satoh K, O'Dell MR, Soe NN, Cui Z, Mohan A, et al. Cyclophilin A is an inflammatory mediator that promotes atherosclerosis in apolipoprotein E-deficient mice. $J$ Exp Med 2011; 208: 53-66.

18. Shimokawa H, Takeshita A. Rho-kinase is an important therapeutic target in cardiovascular medicine. Arterioscler Thromb Vasc Biol 2005; 25: $1767-1775$.

19. Satoh K, Fukumoto Y, Shimokawa H. Rho-kinase: Important new therapeutic target in cardiovascular diseases. Am J Physiol Heart Circ Physiol 2011; 301: H287-H296.

20. Satoh K, Kagaya Y, Nakano M, Ito Y, Ohta J, Tada H, et al. Important role of endogenous erythropoietin system in recruitment of en- dothelial progenitor cells in hypoxia-induced pulmonary hypertension in mice. Circulation 2006; 113: $1442-1450$.

21. Nakayama M, Osaki S, Shimokawa H. Validation of mortality risk stratification models for cardiovascular disease. Am J Cardiol 2011; 108: $391-396$.

22. Pencina MJ, D'Agostino RB Sr, D'Agostino RB Jr, Vasan RS. Evaluating the added predictive ability of a new marker: From area under the ROC curve to reclassification and beyond. Stat Med 2008; 27: 157-172; Discussion 207-212.

23. Satoh K, Nigro P, Zeidan A, Soe NN, Jaffre F, Oikawa M, et al. Cyclophilin A promotes cardiac hypertrophy in apolipoprotein E-deficient mice. Arterioscler Thromb Vasc Biol 2011; 31: 1116-1123.

24. Satoh K, Shimokawa H, Berk BC. Cyclophilin A: Promising new target in cardiovascular therapy. Circ J 2010; 74: 2249-2256.

25. Higashi M, Shimokawa H, Hattori T, Hiroki J, Mukai Y, Morikawa K, et al. Long-term inhibition of Rho-kinase suppresses angiotensin IIinduced cardiovascular hypertrophy in rats in vivo: Effect on endothelial NAD(P)H oxidase system. Circ Res 2003; 93: 767-775.

26. Gao JY, Yasuda S, Tsuburaya R, Ito Y, Shiroto T, Hao K, et al. Longterm treatment with eicosapentaenoic acid ameliorates myocardial ischemia-reperfusion injury in pigs in vivo: Involvement of Rhokinase pathway inhibition. Circ J 2011; 75: 1843-1851.

27. Griendling KK, FitzGerald GA. Oxidative stress and cardiovascular injury. Part II: Animal and human studies. Circulation 2003; 108: 2034-2040.

28. Taniyama Y, Griendling KK. Reactive oxygen species in the vasculature: Molecular and cellular mechanisms. Hypertension 2003; 42: $1075-1081$.

29. Rao GN, Berk BC. Active oxygen species stimulate vascular smooth muscle cell growth and proto-oncogene expression. Circ Res 1992; 70: $593-599$.

30. Baas AS, Berk BC. Differential activation of mitogen-activated protein kinases by $\mathrm{H}_{2} \mathrm{O}_{2}$ and $\mathrm{O}_{2}{ }^{-}$in vascular smooth muscle cells. Circ Res 1995; 77: 29-36.

31. Kikuchi Y, Ito K, Ito Y, Shiroto T, Tsuburaya R, Aizawa K, et al. Double-blind and placebo-controlled study of the effectiveness and safety of extracorporeal cardiac shock wave therapy for severe angina pectoris. Circ J 2010; 74: 589-591.

32. Thomas M, Gavrila D, McCormick ML, Miller FJ Jr, Daugherty A, Cassis LA, et al. Deletion of p47phox attenuates angiotensin IIinduced abdominal aortic aneurysm formation in apolipoprotein E-deficient mice. Circulation 2006; 114: 404-413.

33. Seizer P, Schonberger T, Schott M, Lang MR, Langer HF, Bigalke B, et al. EMMPRIN and its ligand cyclophilin A regulate MT1-MMP, MMP-9 and M-CSF during foam cell formation. Atherosclerosis 2010; 209: $51-57$.

34. Guo H, Majmudar G, Jensen TC, Biswas C, Toole BP, Gordon MK. Characterization of the gene for human emmprin, a tumor cell surface inducer of matrix metalloproteinases. Gene 1998; 220: 99-108.

35. Haug C, Lenz C, Diaz F, Bachem MG. Oxidized low-density lipoproteins stimulate extracellular matrix metalloproteinase inducer (EMMPRIN) release by coronary smooth muscle cells. Arterioscler Thromb Vasc Biol 2004; 24: 1823-1829.

36. Yurchenko V, Zybarth G, O'Connor M, Dai WW, Franchin G, Hao $\mathrm{T}$, et al. Active site residues of cyclophilin A are crucial for its signaling activity via CD147. J Biol Chem 2002; 277: 22959-22965.

\section{Supplementary Files}

Supplementary File 1

Data S1. CyPA and hsCRP

Figure S1. Reproducibility of plasma cyclophilin A (CyPA) measurements in duplicates.

Figure S2. Diagnostic performance of plasma cyclophilin A (CyPA) for coronary disease in patients in the higher and lower high-sensitivity C-reactive protein (hsCRP) subgroups.

Figure S3. Number of stenotic coronary arteries and the requirement for cardiovascular intervention according to quartiles of high-sensitivity C-reactive protein (hSCRP).

Figure S4. Gensini score according to quartiles of cyclophilin A (CyPA) and high-sensitivity C-reactive protein (hsCRP).

Table S1. Baseline Clinical Characteristics According to the hsCRP Levels and the Presence or Absence of Clinically Significant CAD

Please find supplementary file(s);

http://dx.doi.org/10.1253/circj.CJ-12-0805 